SUPPORTING INFORMATION

\title{
A Quantum Dot Nanobiosensor for Rapid Detection of Botulinum Neurotoxin Serotype E
}

Yun Wang, ${ }^{\dagger}$ Kristin M. Schill, ${ }^{\dagger}$ H. Christopher Fry, ${ }^{\ddagger}$ Timothy V. Duncan ${ }^{t, *}$

${ }^{\dagger}$ Center for Food Safety and Applied Nutrition, U.S. Food and Drug Administration,

Bedford Park, IL 60501

${ }^{\ddagger}$ Center for Nanoscale Materials, Argonne National Laboratory, DuPage County, Illinois 60439

*Address correspondence to Timothy.Duncan@fda.hhs.gov 


\section{ADDITIONAL MATERIALS AND METHODS}

Peptide and quantum dot (QD) conjugation. Peptide probe specifically designed for BoNT/E detection was assembled onto QDs through the high affinity of poly-histidine to ITK QDs with amphiphilic block-copolymer-type surfaces in the presence of $\mathrm{Ni}^{2+} .{ }^{1}$ We measured the photoluminescence (PL) intensity of QD655 (QD with emission maxima at $655 \mathrm{~nm}$ ) at different concentrations and decided that 1.2 pmole QD655 (added to each $50 \mu \mathrm{L}$ sample) would be used in the following experiments. Different amounts of peptide ( 0 to 60 pmole) were added to 1.2 pmole QD, giving a peptide vs. QD molar ratio of 0 to 50 , and each solution was incubated for $1 \mathrm{~h}$. PL of samples with and without (0 pmole peptide) was measured. Förster resonance energy transfer (FRET) efficiency $(E)$ was calculated based on the changes in PL as:

$$
E=\frac{P L_{D}-P L_{D A}}{P L_{D}}
$$

where $P L_{D}$ represents the $\mathrm{PL}$ when the quencher is absent (0 pmole of peptide) and $P L_{D A}$ represents the PL in the presence of quencher (peptide). Relative PL was calculated to reveal the changes in $\mathrm{PL}$ with increasing peptide vs. QD ratio as:

$$
\text { Relative } P L=\frac{P L_{D A}}{P L_{D}}
$$

The optimal peptide vs. QD (peptide:QD) molar ratio was determined to be the ratio when the relative $\mathrm{PL}$ is lower than $10 \%$ and the decreasing trend of relative $\mathrm{PL}$ starts levelling off. This ratio was employed in the following experiment. The peptide self-assembly through the polyhistidine sequence onto the QD surface was quick, as also observed in our previous study. ${ }^{2}$ We confirmed this by letting the peptide and QD react for a time length of 0.5 to $4 \mathrm{~h}$ at room temperature. The relative PL was calculated for each reaction time to determine an optimized reaction time that balances effective peptide-QD binding with a reasonable total assay detection time. 


\section{ADDITIONAL RESULTS AND DISCUSSION}

FRET overview. FRET between the peptide and QD was characterized by calculating the theoretical Förster critical distance $\left(R_{0}\right)$, or the distance at which FRET is $50 \%$ efficient, which is estimated using a point-dipole approximation as (in units of $\AA$ ):

$$
R_{0}=0.2108\left(\kappa^{2} \Phi_{D} n^{-4} J_{D A}\right)^{1 / 6}
$$

where $\kappa^{2}$ is the orientation factor (usually approximated as equal to $2 / 3$ ), $\Phi_{D}$ is the quantum yield of the QDs in the absence of quencher (reported by the manufacturer as 0.81 for the QDs used for optimization of the peptide:QD ratio), $n$ is the refractive index of the medium (typically assumed to be 1.4 for aqueous media), and $J_{D A}$ is the spectral overlap integral between donor PL and acceptor absorption: ${ }^{3}$

$$
J_{D A}=\frac{\int_{0}^{\infty} F_{D}(\lambda) \varepsilon_{A}(\lambda) \lambda^{4} d \lambda}{\int_{0}^{\infty} F_{D}(\lambda) d \lambda}
$$

In Eqn. S3B, $F_{D}(\lambda)$ is the unitless donor PL intensity as a function of wavelength $(\lambda)$ in $\mathrm{nm}$ (the denominator normalizes the PL intensity to an area of 1) and $\varepsilon_{A}(\lambda)$ is the molar extinction coefficient of the acceptor in units of $\mathrm{M}^{-1} \mathrm{~cm}^{-1}$.

Calculation of the overlap integral. The detection of BoNT in this study is based on the FRET between the QD and the quencher labeling the peptide. We chose QSY21 quencher as the acceptor because it has an absorption maximum at $660 \mathrm{~nm}$ and has an absorption spectrum that overlaps well with the QD655 emission spectrum, as shown in Figure S1. The absorption of QSY21-labeled peptide (QSY21-pepE) was measured. No significant shift of the QSY21 absorption spectrum was observed during peptide attachment to the QSY21. 


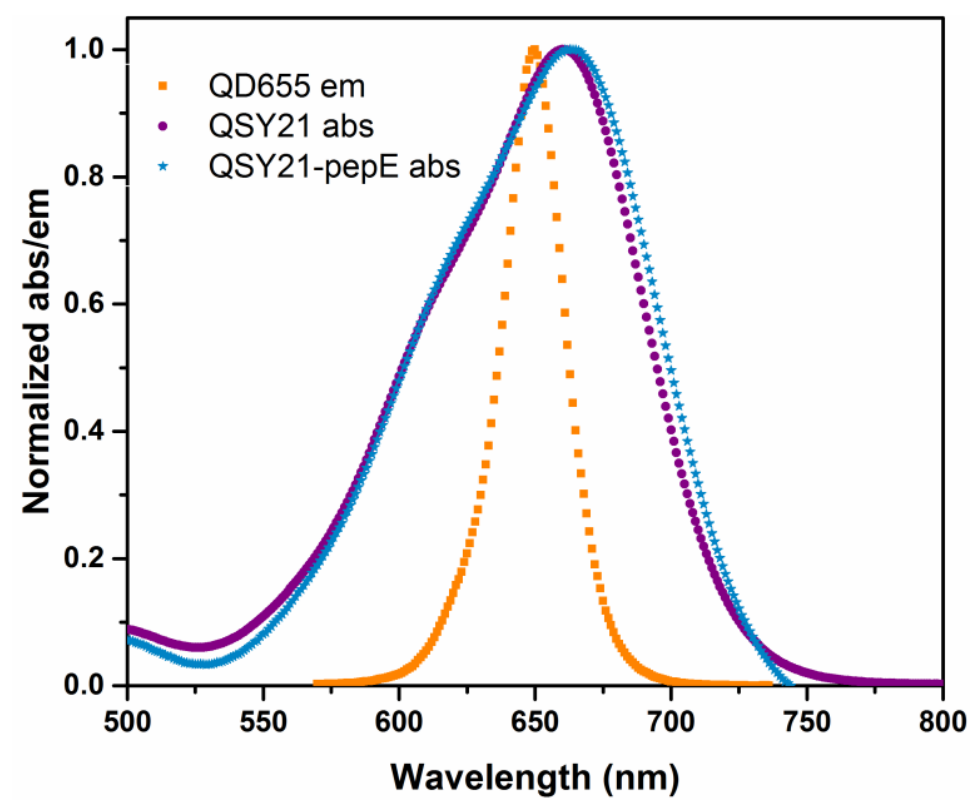

Figure S1. Spectral overlap for the donor (QD655) and acceptor (QSY21) used in the FRETbased nanobiosensor. QSY21 absorption (abs) and QD655 emission (em) are plotted. The absorption of quencher (QSY21) labeled peptide (QSY21-pepE) is plotted as well, and no significant shift in the absorption compared to the quencher in absence of peptide was observed.

Relationships between nanobiosensor signal and BoNT/E holotoxin concentration. The nanobiosensor signal $\left(S / S_{0}\right)$ increases with increasing BoNT/E holotoxin concentration (Figure S2A). A linear relationship between the nanobiosensor signal $\left(S / S_{0}\right)$ and logarithm of BoNT/E concentration was observed over the range of $133 \mathrm{pM}$ to $6.67 \mathrm{nM}$ (Figure S2B). The trend reaches a plateau when BoNT/E concentration reaches $6.67 \mathrm{nM}$ and the $S / S_{0}$ value exceeds 3 , which may be due to cleavage of all the peptide accessible for toxin cleavage during the $2 \mathrm{~h}$ incubation time. The same phenomenon was observed with BoNT/A and /B detection when nanobiosensor signals $\left(S / S_{0}\right)$ reached values exceeding $3 .^{2}$ Although the results imply that the nanobiosensor's quantification capability has an upper limit, the linear range still spans two orders of magnitude. 
A

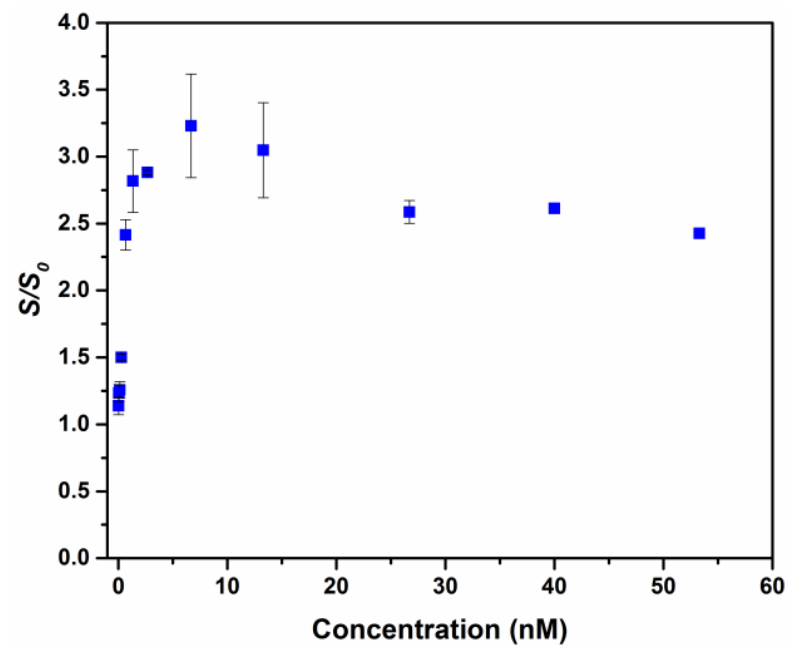

B

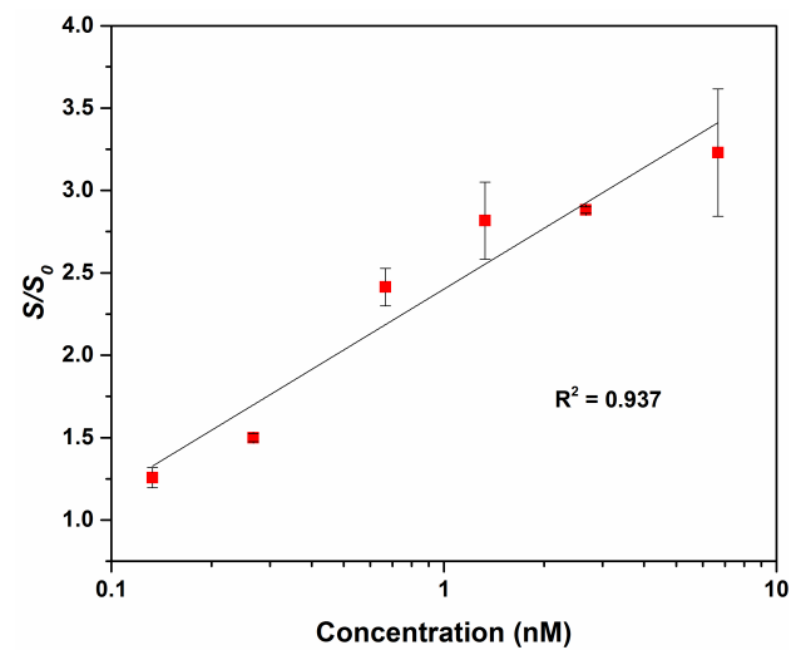

Figure S2. (A) The nanobiosensor signal $\left(S / S_{0}\right.$, PL peak intensity peak of QDs in the presence of QSY21-pepE that was incubated with BoNT/E holotoxin relative to that of the negative control sample) with BoNT/peptide incubation times of $2 \mathrm{~h}$. (B) Linear relationship was found between the nanobiosensor signal and base ten logarithm of the BoNT/E holotoxin concentration in the range of $0.133 \mathrm{nM}$ to $6.67 \mathrm{nM}$, as indicated by the black fit lines. Linear equation: $y=1.22 x+2.40$ ( $\mathrm{y}: \mathrm{S} / \mathrm{S}_{0}$, $\mathrm{x}$ : logarithm of BoNT/E holotoxin concentration in $\mathrm{nM}$ ).

\section{REFERENCES}

1. Yao, H. Q.; Zhang, Y.; Xiao, F.; Xia, Z. Y.; Rao, J. H. Quantum Dot/Bioluminescence Resonance Energy Transfer Based Highly Sensitive Detection of Proteases. Angew Chem Int Edit 2007, 46, 4346-4349.

2. Wang, Y.; Fry, H. C.; Skinner, G. E.; Schill, K. M.; Duncan, T. V. Detection and Quantification of Biologically Active Botulinum Neurotoxin Serotypes A and B Using a Forster Resonance Energy Transfer-Based Quantum Dot Nanobiosensor. ACS Appl Mater Interfaces 2017, 9, 31446-31457.

3. Lakowicz, J. R. Principles of Fluorescence Spectroscopy. 3rd ed.; Springer: New York, 2006; $954 \mathrm{p}$. 